\title{
Resorbable Mesh Cranioplasty Repair of Bilateral Cerebrospinal Fluid Leaks Following Pediatric Simultaneous Bilateral Auditory Brainstem Implant Surgery
}

\author{
*Giacomo Colletti, †Marco Mandalà, ||Vittorio Colletti, §Alberto Deganello, *Fabiana Allevi, \\ and $\ddagger$ Liliana Colletti
}

\begin{abstract}
*Department of Maxillo-Facial Surgery, University of Milan; †Otological and Skull Base Surgery Department, Azienda Ospedaliera Universitaria Senese, Siena; $\ddagger$ ENT Department, University of Verona, Verona; §University of Firenze, Florence; and ||International Center for Performing and Teaching Auditory Brainstem Surgery in Children, Milan, Italy
\end{abstract}

Objective: To present a child with cochlear nerve deficiency (CND) who received simultaneous bilateral simultaneous auditory brainstem implants (BS-ABI) and subsequently presented with bilateral cerebrospinal fluid (CSF) leaks unresponsive to standard treatments. To propose a novel rigid retrosigmoid cranioplasty for treating and preventing CSF leaks in children at high risk for this complication.

Patient: A 3.5-year-old child with CND, vertebral defects, anal atresia, cardiac defects, tracheo-esophageal fistula, renal anomalies, and limb abnormalities, coloboma, heart defect, atresia choanae, retarded growth and development, genital abnormality, and ear abnormality, Arnold Chiari malformation, previous treated tracheo-esophageal fistula underwent BS-ABI. Postoperatively, the child had recurrent bilateral retroauricular fluid collections. A standard revision procedure revealed breaches in the dural closure, migration of the auditory brainstem implantation (ABI) receiver stimulator on both sides and was unsuccessful in stopping the leak.

Interventions: Bilateral repair with free fat grafting filling the craniectomy space and two absorbable meshes of poly-L-
D-lactic (PLDL) acid stabilized with PLDL pins on the surrounding cranium, one to stabilize the fat graft and one to fix the ABI receiver stimulators inside the subperiosteal pockets.

Main Outcome Measure: CSF leak recurrence, postoperative computed tomographic (CT) scans, intra- and postoperative simultaneous electrically evoked auditory brainstem responses (EABRs). Subjective and objective assessment of ABI function.

Results: No postoperative CSF leaks at 60 days follow-up. EABRs and consistent behavioral responses obtained at initial mapping on both sides.

Conclusions: The use of BS-ABI likely contributed to bilateral CSF leaks requiring revision surgeries in this child. Simultaneous bilateral craniotomies can put patients at risk for CSF leak. A novel cranioplasty technique employed finally proved successful in stopping the CSF leak in this case. Key Words: Cranioplasty-CSF leak-Growing patients reconstruction-Retrosigmoid access.

Otol Neurotol 38:606-609, 2017.

\section{OBJECTIVE}

Auditory brainstem implantation (ABI) recently indicated for deaf children unsuitable for cochlear implants (CI) 8 (1-6). Unlike CI, ABI carries the risks of a retrosigmoid surgery (RSS). Cerebrospinal fluid (CSF) collections are rare in children fitted with ABIs (7). When they occur, treatment is needed to avoid meningitis and rarely death. In adults, CSF leakage after RSS has an average incidence of $10.6 \%(8)$ with a range from $0.29(9)$

Address correspondence and reprint requests to Giacomo Colletti, M.D., Department of Maxillo-Facial Surgery, University of Milan, Milan, Italy; E-mail: giacomo.colletti@gmail.com

Disclosure of funding: None.

The authors disclose no conflicts of interest.

Supplemental digital content is available in the text.

DOI: 10.1097/MAO.0000000000001338 to $68.7 \%(10)$. In vestibular schwannoma surgery, the incidence is higher, from 2 to $14 \%$, compared with vestibular nerve section and microvascular decompression procedures which present an average incidence of $6.15 \%(11)$.

CSF leaks after RSS may result from exposure of air cells around the sigmoid during craniotomy, alteration of CSF absorption with increased intracranial pressure associated with blood clots in the cerebellopontine angle and prolonged surgical time. In the present case, the additional presence of an $\mathrm{ABI}$ electrode cable perforating the dural closure increased the risk of CSF flow from the intradural space to the subcutaneous plane. Additionally, the use of simultaneous bilateral craniotomies can put patients at higher risk for CSF leak given the inability to provide pressure to the wound during second sided RSS. 
Since 2000, the 103 children implanted worldwide with $\mathrm{ABI}$ by the senior surgeon presented an overall incidence of postoperative CSF leaks of $9.9 \%$ for primary surgeries. This figure compares favorably with our $11.8 \%$ overall CSF leak rate in 459 total primary RSS performed during the same period.

Recently, we realized that children implanted with ABI with upper respiratory (UR) issues, presented a higher prevalence of CSF leak and were at greater risk of revisions, compared with children that did not have UR issues. These observations motivated a change in our retrosigmoid cranioplasty procedure that included two absorbable meshes of poly-L-D-lactic (PLDL) acid, fixed with PLDL pins on the surrounding cranium to stabilize the fat graft and the ABI receiver stimulator. This novel cranioplasty decreased our percentage of CSF leaks from 9.9 (10 of 103) in ABI children and 11.8 (54 of 459) in total primary RSS to $1.3 \%$ ( 1 of 76) in all RSS performed after the adoption of the new technique.

The case described in the present paper refers to a child with bilateral simultaneous $\mathrm{ABI}$ (BS-ABI), originally in the cohort of standard cranioplasty and after unsuccessful revision surgery for CSF leak was included in the cohort of the new cranioplasty technique.

\section{PATIENT}

A 3.5-year-old boy, with bilateral cochlear and cochlear nerve aplasia, Arnold Chiari, coloboma, heart defect, atresia choanae, retarded growth and development, genital abnormality, and ear abnormality, vertebral defects, anal atresia, cardiac defects, tracheo-esophageal fistula, renal anomalies, and limb abnormalities/VATER syndromes, and corrected trachea esophageal fistula, was referred to our pediatric $\mathrm{ABI}$ Center to undergo BS-ABI. He had a history of recurrent chronic wet cough with frequent paroxysmal coughing spells and obstructive sleep apnea (OSA), which the anesthesiologist did not consider to be a contraindication for ABI. After discussing the risks and benefits of ABI surgery with the parents, they clearly selected the BS-ABI procedure, opting for the Medel Opus II (MED-EL Elektromedizinische Geräte GmbH, Innsbruck, Austria) ABI device. Indeed, the parents knew that our Center had previously performed with success both sequential and single stage BS-ABI in NF2 adults and in children with cochlear nerve deficiency (CND).

\section{Intervention}

Single sided ABI setup was performed using our standard protocol (11); however, a modified technique was employed for bilateral simultaneous implantation with the child in the lateral position and both ears simultaneously prepped/draped. Surgery started on the right side. The receiver-stimulator (RS) was placed in a subperiosteal pocket with no fixation and, after positioning the $\mathrm{ABI}$ array on the cochlear nuclei evoked auditory brainstem responses (EABRs) were obtained from all 12 electrodes. The dura and the cable at the "exit/entry"

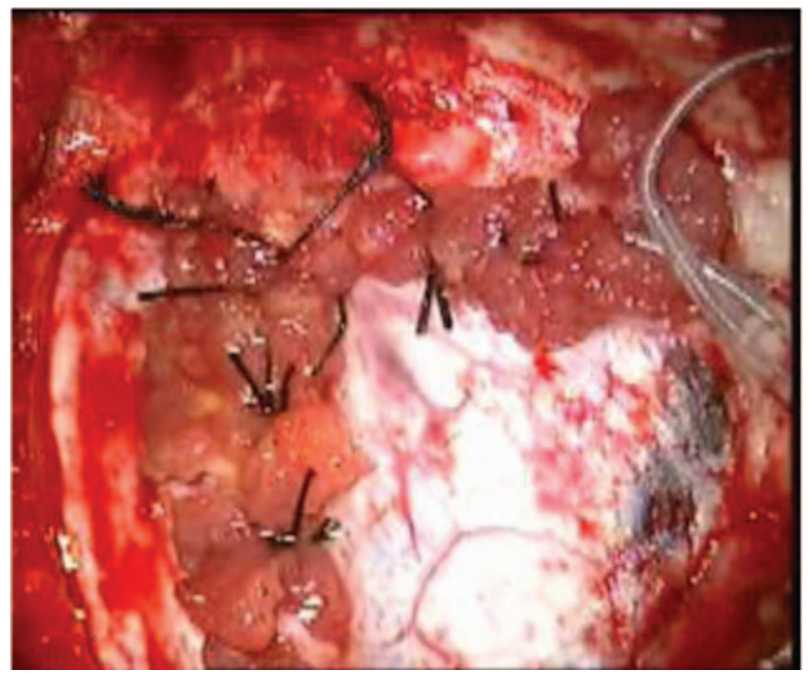

FIG. 1. The dura was closed in a watertight fashion with muscle strips continuously running from inside to outside of the stiches of the dura and around the implant lead.

zone of the dura was closed according to our standard procedure (Fig. 1). The cavity of retro sigmoid cranioplasty (RSC) filled with fat graft was covered by a large "Palva" flap and the overlying tissue closed in layers.

The compression garment was limited to the right retroauricular area with an open window (Supplemental figure, http://links.lww.com/MAO/A495) on the RS area for placing the SP and verifies whether ongoing $\mathrm{ABI}$ surgery on the left side could induce changes in the EABRs of the previously implanted ABI.

Surgery on the left side followed the same procedure. At the end of surgery, EABRs were obtained simultaneously from both sides. To reduce the risk of swelling and CSF leaks a new compression garment was applied bilaterally and left in place for 72 hours.

Bulky CSF collections became evident postoperatively on day 6 and 8 , respectively in the left and right retroauricular area (Fig. 2). A lumbar drain was not placed because of Arnold Chiari malformation.

\section{REVISION WITH STANDARD PROCEDURE}

Revision showed the RSs migrated from the subperiosteal pocket to the craniectomy area and several large breaches of the dural suture with pulsating CSF flowing out of the breaches. The dura and the cable at the "exit/ entry" zone of the dura were closed according to our standard procedure (Fig. 1). The cavity of RSC filled with fat graft was covered by a large "Palva" flap and the overlying tissue closed in layers.

After 2 to 3 weeks an atypical alternating pattern of decreasing and increasing wound swelling appeared on both operative sites, clearly indicating an inadequacy of our standard RSC for this particular child with UR issues. This induced us to use for this child the recently adopted novel rigid cranioplasty indicated for children with recurrent CSF leaks surgery after ABI surgery. 


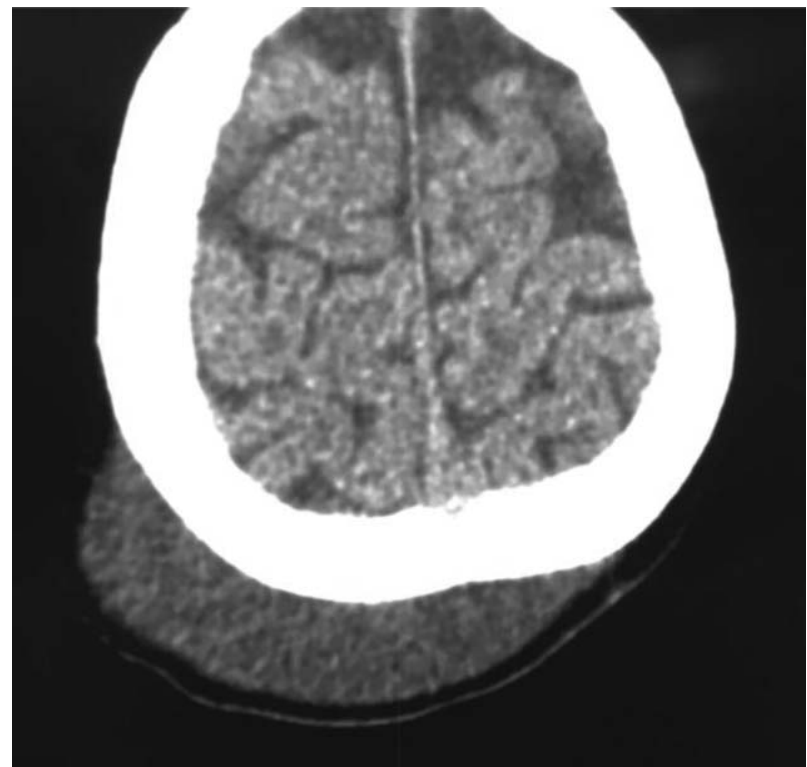

FIG. 2. A bulky CSF collection became evident postoperatively on day 8 on the right retroauricular area. CSF indicates cerebrospinal fluid.

\section{REVISION WITH THE NEW CRANIOPLASTY}

Dural breaches and cables were fixed following our standard procedure. No displacement of ABI cable and paddle were observed as confirmed by EABRs. A fat graft with a thickness equivalent to that of the craniectomy space was positioned over the dura and covered with a thick and large "Palva" flap. To stabilize the fat graft, a rigid cranioplasty was performed with a modeled resorbable mesh of poly-L-D-lactic (PLDL) acid. A smaller resorbable mesh was placed over the RSs inside the subperiosteal pocket. Both meshes were fixed with PLDL pins on the surrounding cranium (Fig. 3). The muscles, subcutaneous tissues, and skin were closed in layers. A soft compressive garment was applied on both retroauricular areas avoiding the area of the RS.

\section{MAIN OUTCOMES MEASURES}

Postoperative computed tomographic (CT) scans excluded complications, confirmed the correct position of both the ABI arrays inside the forth ventricle and showed a reconstruction of the craniotomy morphological similar to the preoperative shape of the bone (Fig. 4). No postoperative CSF leaks were observed at 60 days follow-up. Subjective and objective assessment of ABI functioning was verified at $\mathrm{ABI}$ activation.

Initial mapping on both sides showed bilateral stable postoperative EABRs with consistent behavioral responses.

\section{CONCLUSION}

Bilateral simultaneous ABI likely increased the risk for CSF leak for this child and necessitated two subsequent repair procedures. Fortunately, a modified

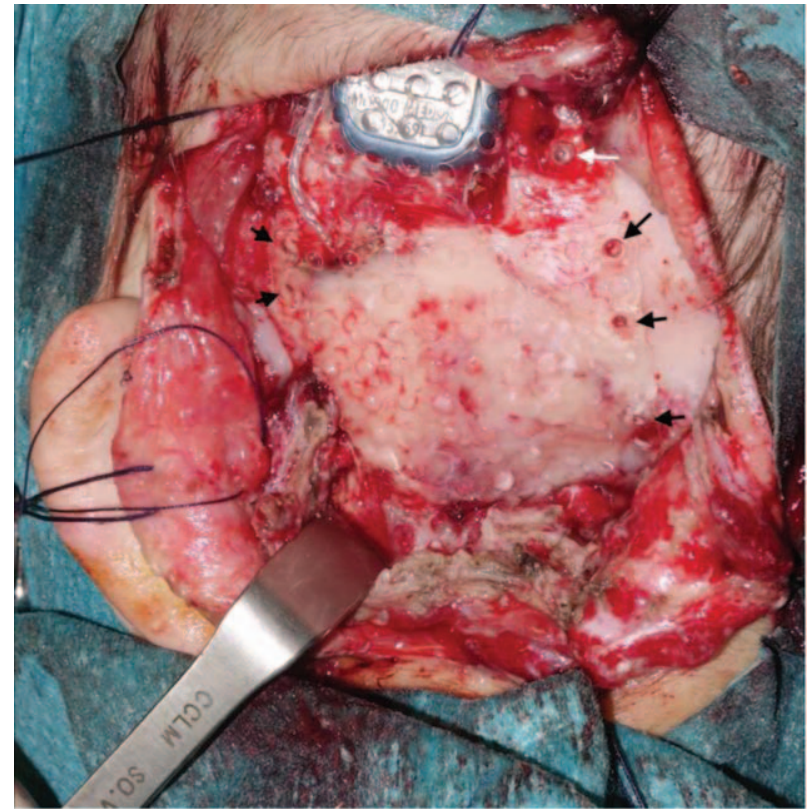

FIG. 3. Rigid cranioplasty performed with a modeled resorbable mesh of poly-L-D-lactic (PLDL) acid. The meshe was fixed with PLDL pins on the surrounding cranium (black arrows).

technique using a fat graft and absorbable miniplate was successful in stopping the leak at 60 days follow-up. In children with CND submitted to ABI without associated UR issues, the relatively low pressure normally exerted

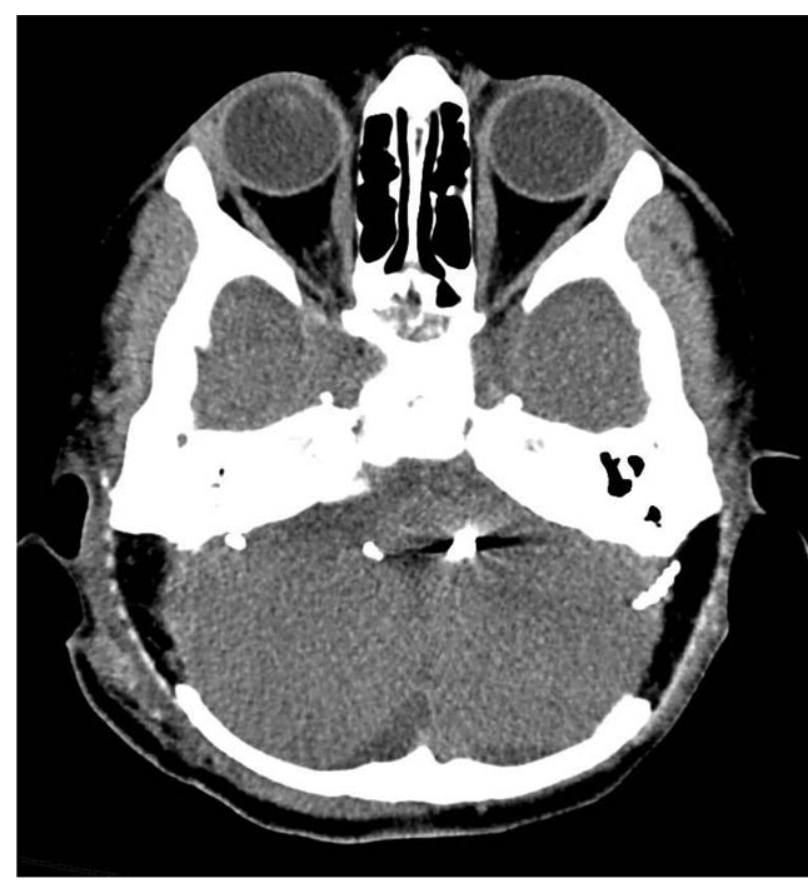

FIG. 4. The postoperative CT scans excluded complications, confirmed the correct position of both the $\mathrm{ABI}$ arrays and showed a morphological reconstruction closely resembling the preoperative shape of the bone. $\mathrm{ABI}$ indicates auditory brainstem implantation; CT, computed tomography. 
by the CSF on the dural sutures, coupled with the particular vascularity of the dura allow for robust wound healing, and leading to a successful closure with no CSF collection in the vast majority of cases, even with standard surgical techniques.

Conversely, children with significant UR issues and OSA often with secondary intracranial hypertension (SIH) (12-16), must be considered at higher risk for a CSF leak postoperatively. Indeed, this particular cohort of children need an individualized assessment of their respiratory problems with emphasis on identifying significant comorbidities responsible for idiopathic intracranial hypertension (IIH) or secondary intracranial hypertension (SIH) before the decision for $\mathrm{ABI}$ is confirmed.

Furthermore, to reduce the risk of CSF leak after surgery, an appropriate modification of the standard surgical strategy of craniectomy repair needs to be adopted in these children. Fat graft alone, effective in repairing most cases of CSF leak (17), needs to be supplemented by a rigid cranioplasty (RCPL) in children with UR issues. RCPL realizing a moderate but rigid, extrinsic counteractive compression on the dura, neutralizes the effects of both the IIH and SIH and the intense and impulsive increased hydrostatic forces produced by chronic coughing.

The present study has the limitation of being a single case report but, to the best of our knowledge, this is also the first description of a BS-ABI in a child with associated UR issues.

Among the 103 children implanted with ABIs by our team since 2000, six were bilateral, four sequential, and two simultaneous. Our experience from these few preliminary children supports further application of bilateral electric stimulation from ABIs for children with CND as now routinely performed for cochlear implantation. Bilateral simultaneous ABI surgery likely increases the risk of CSF leak in patients. Surgeons should be extremely cautious regarding recommending bilateral simultaneous surgery and consider sequential implantation if binaural stimulation is sought.

A review of the pediatric $A B I$ literature reveals insufficient data on the causes and rates and CSF leak to allow a statistical comparison with our data.

Finally, according to our experience the compression garment should be light on the areas where the RSCs are located to avoid ischemia and necrosis on the overlying tissues and very tight all around the head and down to the neck for the first 3 days after surgery. On the fourth day the compression garment should be removed to inspect the wound and then replaced in situ for 4 more days before the child undergoes x-ray of the skull and then dismissed.
The novel rigid RSC demonstrated effectiveness in the reported child fitted with BSABIs with recurrent CSF leaks. It is presently systematically used in our pediatric $\mathrm{ABI}$ surgery protocol to minimize the incidence of postoperative CSF collections.

\section{REFERENCES}

1. Colletti V, Fiorino F, Sacchetto L, Miorelli V, Carner M. Hearing habilitation with auditory brainstem implantation in two children with cochlear nerve aplasia. Int $J$ Pediatr Otorhinolaryngol 2001;60:99-111.

2. Colletti V, Fiorino FG, Carner M, Miorelli V, Guida M, Colletti L. Auditory brainstem implant as a salvage treatment after unsuccessful cochlear implantation. Acta Otolaryngol 2004;124:353-7.

3. Colletti L. Beneficial auditory and cognitive effects of auditory brainstem implantation in children. Acta Otolaryngol 2007;127: 943-6.

4. Colletti L, Wilkinson EP, Colletti V. Auditory brainstem implantation after unsuccessful cochlear implantation of children with clinical diagnosis of cochlear nerve deficiency. Ann Otol Rhinol Laryngol 2013;122:605-12.

5. Colletti L, Shannon RV, Colletti V. The development of auditory perception in children after auditory brainstem implantation. Audiol Neurootol 2014;19:386-94.

6. Sennaroglu L, Ziyal I, Atas A, et al. Preliminary results of auditory brainstem implantation in prelingually deaf children with inner ear malformations including severe stenosis of the cochlear aperture and aplasia of the cochlear nerve. Otol Neurotol 2009;30:708-15.

7. Colletti V, Shannon RV, Carner M, Veronese S, Colletti L. Complications in auditory brainstem implant surgery in adults and children. Otol Neurotol 2010;31:558-64.

8. Selesnick SH, Liu JC, Jen A, Newman J. The incidence of cerebrospinal fluid leak after vestibular schwannoma surgery. Otol Neurotol 2004;25:387-93.

9. Park JS, Kong DS, Lee JA, Park K. Intraoperative management to prevent cerebrospinal fluid leakage after microvascular decompression: dural closure with a "plugging muscle" method. Neurosurg Rev 2007;30:139-42.

10. Bayazit YA, Celenk F, Duzlu M, Goksu N. Management of cerebrospinal 185 fluid leak following retrosigmoid posterior cranial fossa surgery. ORL J Otorhinolaryngol Relat Spec 2009; 71:329-33.

11. Colletti V, Fiorino FG, Carner M, Giarbini N, Sacchetto L, Cumer $\mathrm{G}$. The retrosigmoid approach for auditory brainstem implantation. Am J Otol 2000;21:826-36.

12. Quinn AG, Gouws P, Headland S, et al. Obstructive sleep apnea syndrome with bilateral papilledema and vision loss in a 3-year-old child. J AAPOS 2008;12:197-9.

13. Hanigan WC, Zallek SN. Headaches, shunts, and obstructive sleep apnea: report of two cases. Neurosurgery 2004;54:764-8.

14. Marcus DM, Lynn J, Miller JJ, Chaudhary O, Thomas D, Chaudhary B. Sleep disorders: a risk factor for pseydotumor cerebri. $J$ Neuroophthalmol 2001;21:121-3.

15. Lee A. Pseudotumor and sleep apnea. $J$ Neuroophthalmol 2001;21:235.

16. Wall M, Purvin V. Idiopathic intracranial hypertension in men and the relationship to sleep apnea. Neurology 2009;72:300-1.

17. Lüdemann WO, Stieglitz LH, Gerganov V, Samii A, Samii M. Fat implant is superior to muscle implant in vestibular schwannoma surgery for the prevention of cerebrospinal fluid fistulae. Neurosurgery 2008;63:ONS38-42. 